\title{
Effect of enteral nutrition on plasma soluble adhesion molecules in an elderly population
}

\author{
J. Olza ${ }^{1}$, M. D. Mesa ${ }^{1}$, R. Moreno ${ }^{2}$, A. Pérez de la Cruz ${ }^{2}$, A. Gil ${ }^{1}$ and C. M. Aguilera ${ }^{1}$ \\ ${ }^{1}$ Institute of Nutrition and Food Technology, Dept Biochemistry and Molecular Biology II, University of Granada, Granada, \\ Spain and ${ }^{2}$ Universitary Hospital Virgen de las Nieves, Unit of Clinical Nutrition and Dietetic, Granada, Spain
}

The entry of inflammatory cells into the arterial wall is mediated by adhesion molecules expressed on the endothelial surface by binding to their counter-ligands on the leucocytes. The origin of soluble adhesion molecules such as endothelial selectin (sE-selectin), intercellular adhesion molecule 1 (sICAM-1) and vascular cell adhesion molecule 1 (sVCAM-1) is mainly the activated endothelium. Thus, they are considered to be biomarkers of the inflammatory state of the arterial wall ${ }^{(1)}$. sICAM-1 appears to be a general marker of a proinflammatory status, whereas SVCAM-1 emerges as a predictor for future cardiovascular events in patients with pre-existing disease. Both DHA and EPA have been found to decrease the expression of soluble adhesion molecules in studies of the cytokine-activated endothelium in vitro ${ }^{(2)}$. Enteral nutrition may influence inflammatory molecule secretion in patients under pathological conditions. Feeding long-chain $n$-3 PUFA results in partial replacement of arachidonic acid in cell membranes by EPA. A number of experimental and human trials have demonstrated immunomodulatory properties of $n$-3 PUFA, by suppressing different variables of immune function, including the production of inflammatory cytokines and eicosanoids ${ }^{(3)}$. There are no reports of studies that have evaluated the influence of dietary EPA and DHA on soluble adhesion molecules in elderly patients fed an enteral diet, which is the aim of the present study.

Thirty-two patients aged 75 years were fed a complete formula for enteral nutrition (T-Diet Plus ${ }^{\circledR}$; Vegenat SA, Madrid, Spain) with added EPA ( $75 \mathrm{mg} / \mathrm{l})$ and DHA $(35 \mathrm{mg} / \mathrm{l})$, as compared with standard diets. Patients were monitored for 6 months, and blood samples were taken at the beginning and after 3 and 6 months of feeding. At the end of the experimental period only sixteen patients remained in the study. Mean daily intake was 5459 (SE 130) kJ/d. Plasma EPA and DHA were measured by GC in order to establish fatty acid incorporation; EPA increased by approximately $41 \%$ while DHA increased by $6 \%$. The plasma soluble adhesion molecules sE-selectin, sICAM-1 and sVCAM-1 were measured by immunoassay with a LINCOplex ${ }^{\mathrm{TM}}$ kit (Millipore Corp., Billerica, MA, USA) using the Luminex 200 System built on xMAP technology (Luminex Corp., Austin, TX, USA). A non-parametric Wilcoxon test was used to determine statistical differences between dietary interventions after 3 or 6 months $(P \leq 0.05)$.

\begin{tabular}{|c|c|c|c|c|c|c|}
\hline \multirow[b]{2}{*}{ T-Diet Plus ${ }^{\circledR}$} & \multicolumn{2}{|c|}{ sE-selectin $(\mathrm{ng} / \mathrm{ml})$} & \multicolumn{2}{|c|}{ sICAM-1 (ng/ml) } & \multicolumn{2}{|c|}{ sVCAM-1 (ng/ml) } \\
\hline & Mean & $\mathrm{SE}$ & Mean & $\mathrm{SE}$ & Mean & $\mathrm{SE}$ \\
\hline Initial & 35.0 & 3.37 & 238.9 & 29.0 & 1161 & 91.8 \\
\hline 3 month & 34.0 & 2.34 & 254.6 & 34.8 & 1134 & 70.2 \\
\hline 6 month & 29.5 & 3.87 & 286.6 & 59.3 & 1288 & 90.4 \\
\hline
\end{tabular}

sE-selectin tended to decrease over the 6-month period after dietary intervention, although differences were not significant, probably because of the great variation between patients with a number of underlying pathologies. sICAM-1 and sVCAM were not modified after the experimental period. The decrease in some plasma adhesion molecules may indicate an improved outcome for elderly patients fed enteral diets, which may have the potential to be complementary to pharmacological treatments.

This study was financed by Vegenat SA.

1. Paoletti R, Gotto AM Jr \& Hajjar DP (2004) Circulation 109, 20-26.

2. Yaqoob P \& Calder P (2003) Eur J Med Res 8, 337-354.

3. Mesa MD, Aguilera CM \& Gil A (2006) Nutr Hosp 21, 105-118. 